\title{
Radiation-stimulated relaxation of internal mechanical straines in homoepitaxial GaP films
}

\author{
P.A. Gentsar, A.A. Kudryavtsev \\ V. Lashkaryov Institute of Semiconductor Physics, NAS of Ukraine, 45, prospect Nauki, 03028 Kyiv, Ukraine \\ E-mail: journal@isp.kiev.ua
}

\begin{abstract}
The electroreflectance method based on the electrolyte technique is used for investigation of electron transitions $E_{0}, E_{0}+\Delta_{0}$ in homoepitaxial films $n$-GaP (111) with the electron concentration $5.7 \cdot 10^{23} \mathrm{~m}^{-3}$ before and after irradiation by ${ }^{60} \mathrm{Co}$ gamma quanta in the dose range $10^{5}-10^{6} \mathrm{rad}$ under the room temperature. The authors observed splitting the low-energy extremum after irradiation. The decrease in internal mechanical strains inside the films as a result of gamma irradiation was estimated via changes of the electron transition energy and collision parameter of widening. Also estimated is the time of charge carrier energy relaxation after irradiation.
\end{abstract}

Keywords: electroreflectance, gallium phosphide, homoepitaxial film.

Paper received 23.03.04; accepted for publication 21.10.04.

Heteroepitaxial deposition of semiconductor films is widely used in currently electronics and physical investigations. And the characteristic feature of heterosystems obtained in this way is the presence of internal mechanical straines in them.

These straines arising due to some mismatch between lattice constants of the film and substrate as well as differences of their thermal expansion coefficients result in various effects: from changes of the energy band structure up to appearance of technological defects at interfaces [1].

Their changes in the course of semiconductor device exploitation reduce the operation stability of these devices [2]. However, internal mechanical straines can also arise in homoepitaxial systems being caused by a difference in the charge carrier concentrations inherent to the film and substrate [3].

The modulation electroreflectance spectroscopy plays an important role in investigations of the band structure in solids, which stems from its high resolution ability. In comparison with the classical spectroscopy, the electroreflectance one is more sensitive to changes in the semiconductor energy spectrum [4,5]. In this report, the electroreflectance method is used to study the impact of gamma irradiation on internal straines in homoepitaxial gallium phosphide films.

We studied electroreflectance spectra of homoepitaxial $n$-GaP (111) films with the electron concentration
$5.7 \cdot 10^{23} \mathrm{~m}^{-3}$ before and after irradiation with ${ }^{60} \mathrm{Co}$ gamma quanta within the dose range $10^{5}-10^{6} \mathrm{rad}$. The films of the thickness $10^{-7} \ldots 5 \cdot 10^{-6} \mathrm{~m}$ were prepared using the gasphase epitaxy on $n$-GaP substrates with the electron concentration $10^{24} \mathrm{~m}^{-3}$.

The electroreflectance signal was measured under the room temperature using the electrolyte technique (electrolyte was water solution of $1 \mathrm{~N} \mathrm{KCl}$ ) and the first modulation harmonic frequency $2.2 \mathrm{kHz}$ with the threshold sensitivity $5 \cdot 10^{-6}$ and spectral resolution $0.003 \mathrm{eV}$.

The measurements were performed within the spectral range $2.5-3.2 \mathrm{eV}$ including the direct transitions $E_{0}\left(\Gamma_{8 \mathrm{~V}}-\Gamma_{6 \mathrm{C}}\right)$ and $E_{0}+\Delta_{0}\left(\Gamma_{7 \mathrm{~V}}-\Gamma_{6 \mathrm{C}}\right)$ in non-polarized light, since the polarization dependence of electroreflectance is absent in the case of (111) surface.

Shown in Fig. 1 are electroreflectance spectra of films before (curve 1) and after (curve 2) irradiation with gamma quanta by using the dose $2 \cdot 10^{5} \mathrm{rad}$. To avoid the effect of field widening, our spectral measurements were performed applying the same electric fields to the samples (the constant bias was equal to $-0.6 \mathrm{~V}$ ).

The spectrum of non-irradiated film (curve 1) consists of two peaks: negative and positive ones. A high level of mechanical straines at the heterointerface and inside the film resulted in tailing the spectrum.

The electron transitions $E_{0}$ with the energy $2.74 \mathrm{eV}$ and $E_{0}+\Delta_{0}$ with the energy $2.84 \mathrm{eV}$ of GaP [6] remain unresolved here. In this case, the forbidden gap width $E_{0}$ 


\section{P.A. Gentsar, A.A. Kudryavtsev: Radiation-stimulated relaxation of internal ...}

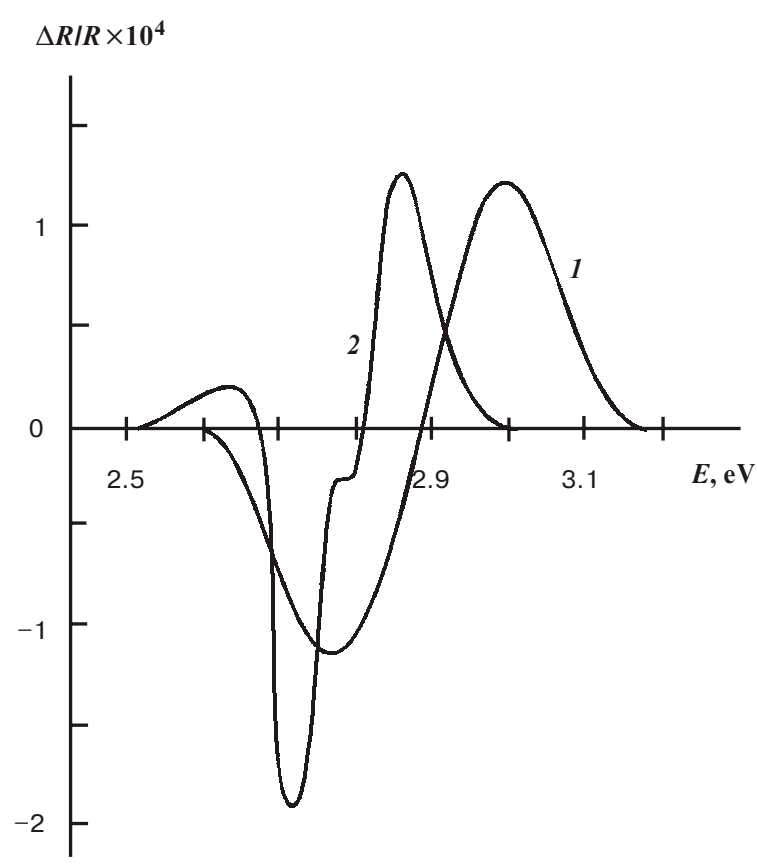

Fig. 1. Electroreflectance spectra of $n-\mathrm{GaP}$ (111) film before (1) and after (2) irradiation by $\gamma$-guanta with the dose $2 \cdot 10^{5} \mathrm{rad}$. The constant bias is $-0.6 \mathrm{~V}$. The modulating voltages are: $1-0.9 ; 2-$ $0.5 \mathrm{~V}$. The flat-band potential is equal to $2.1 \mathrm{~V}$.

was determined using the value of the first extremum in the spectrum, while the collision parameter of widening $\Gamma$ was determined via the halfwidth of this peak. The obtained $E_{0}$ and $\Gamma$ values for the non-irradiated sample were 2.772 and $0.146 \mathrm{eV}$, respectively.

After irradiation of the film with the dose $2 \cdot 10^{5} \mathrm{rad}$ (curve 2), mechanical straines were reduced, the system approached to its structurally equilibrium state. There were observed an increase in peak amplitudes and narrowing the spectral band.

It should be noted that the curve 1 was obtained under the modulation voltage $0.9 \mathrm{~V}$ and the curve 2 under $0.5 \mathrm{~V}$. As known, under increasing modulation voltage, the peak amplitude should increase [4]. In our experiments, we observed the higher signal amplitude for the lower modulation voltage (curve 2).

When the electric field in the depletion layer of the irradiated film is increased (constant bias is equal to $-0.2 \mathrm{~V}$ ), the peak amplitude rises more and more (Fig. 2). Besides, the energy positions of peaks in the electroreflectance spectrum and separation between them are changed, too. After irradiation of the films, $E_{0}$ peak shifted by the value $\Delta E_{0}=0.043 \mathrm{eV}$ into the lower energy side (Fig. 1, curve 2).

It implies that the initial homoepitaxial system possessed internal mechanical straines of the compression type.

Under the hydrostatic pressure $P$, the change in the transition energy is $\Delta E_{0}=\frac{\left(D_{C}-D_{V}\right) \Delta V}{V}$, where $D_{C}$ and
$D_{V}$ are deformation potentials of the conduction and valence band, respectively [7]; $\Delta V / V=-3 P\left(S_{11}+2 S_{12}\right)$ is the relative volume change, where $\left(S_{11}+2 S_{12}\right)^{-1}=$ $C_{11}+2 C_{12}$.

$$
\text { Then, } \Delta E_{0} / P=\frac{-3\left(D_{C}-D_{V}\right)}{\left(C_{11}+2 C_{12}\right)} \text {. For GaP: }\left(D_{C}-D_{V}\right)=
$$

$=-9.3 \mathrm{eV} ; C_{11}=1.4^{12} \cdot 10^{11} \mathrm{~Pa} ; C_{12}=0.625 \cdot 10^{11} \mathrm{~Pa}$, where $C_{11}$ and $C_{12}$ are elastic moduli [5]. Thereof, one can get the value of the coefficient of GaP transition energy change caused by the applied hydrostatic pressure $\Delta E_{0} / \Delta P=1.05 \cdot 10^{-10} \mathrm{eV} / \mathrm{Pa}$.

The estimation of the change in the value of internal mechanical straines in GaP film when $\Delta E_{0}=0.043 \mathrm{eV}$ gives the value $\Delta P=4.1 \cdot 10^{8} \mathrm{~Pa}$.

In the case of uniaxial compression in [111] direction, $\frac{\Delta E_{0}}{P}=\frac{1}{\sqrt{3}} d S_{44}=\frac{1}{\sqrt{3}} d \frac{1}{C_{44}}$, where $d$ is the shear deformation potential; $C_{44}$-elastic modulus $(d=-4.5 \mathrm{eV}$, $C_{44}=0.705 \cdot 10^{11} \mathrm{~Pa}$ for GaP) [5]. Then, $\Delta E_{0} / \Delta P=$ $=0.369 \cdot 10^{-10} \mathrm{eV} / \mathrm{Pa}$, and the change of internal mechanical straines with $\Delta E_{0}=0.043 \mathrm{eV}$ is $11.7 \cdot 10^{8} \mathrm{~Pa}$ as a result of gamma irradiation.

Consequently, under gamma ray action with the dose value $2 \cdot 10^{5} \mathrm{rad}$, the mechanical straines in GaP film were decreased by the value averaged between the values $4.1 \cdot 10^{8} \mathrm{~Pa}$ (hydrostatic pressure) and $11.7 \cdot 10^{8} \mathrm{~Pa}$ (uniaxial compression in [111] direction), that is by $7.9 \cdot 10^{8} \mathrm{~Pa}$ (biaxial compression).

When applying the constant bias $-0.2 \mathrm{~V}$ to irradiated samples, except the increase of the amplitude in spectra, we can observe more clear spectral resolution of $E_{0}$ and $E_{0}+\Delta_{0}$ transitions.

It is clearly seen from Fig. 2 that the low-energy extremum is splitted due to the reduced scattering of charge carriers, and owing to the fact the amplitude of the electroreflectance signal increases as caused by transitions from the spin-orbitally off-splitted valence band.

The difference between energies of the main and offsplitted peaks in Fig. 2 corresponds to the value of the spin-orbital splitting $\Delta_{0}=\Gamma_{8 v}-\Gamma_{7 v}=0.11 \mathrm{eV}$, which is in good agreement with the value $0.10 \mathrm{eV}$ given in [6].

The decrease of the collision parameter of widening $\Gamma$ after irradiation from 0.146 down to $0.080 \mathrm{eV}$ is indicative of the increasing time of charge carrier energy relaxation $\tau=\hbar / \Gamma$ from $4.5 \cdot 10^{-15}$ up to $8.2 \cdot 10^{-15}$ s and the tendency of the initial homoepitaxial system with mechanical straines to approach to its structurally equilibrium state.

With increasing the irradiation dose up to $10^{6} \mathrm{rad}$, the transition energies were practically not changed, while the widening parameter was slightly decreased.

Thus, the analysis of the obtained electroreflectance spectra of $n$-GaP homoepitaxial films has shown that the films grown by using gas-phase epitaxy possess rather high internal mechanical compression straines.

Under $2 \cdot 10^{5} \mathrm{rad}$ gamma irradiation action, these were decreased by the value $7.9 \cdot 10^{8} \mathrm{~Pa}$ as a result of radia- 


\section{P.A. Gentsar, A.A. Kudryavtsev: Radiation-stimulated relaxation of internal ...}

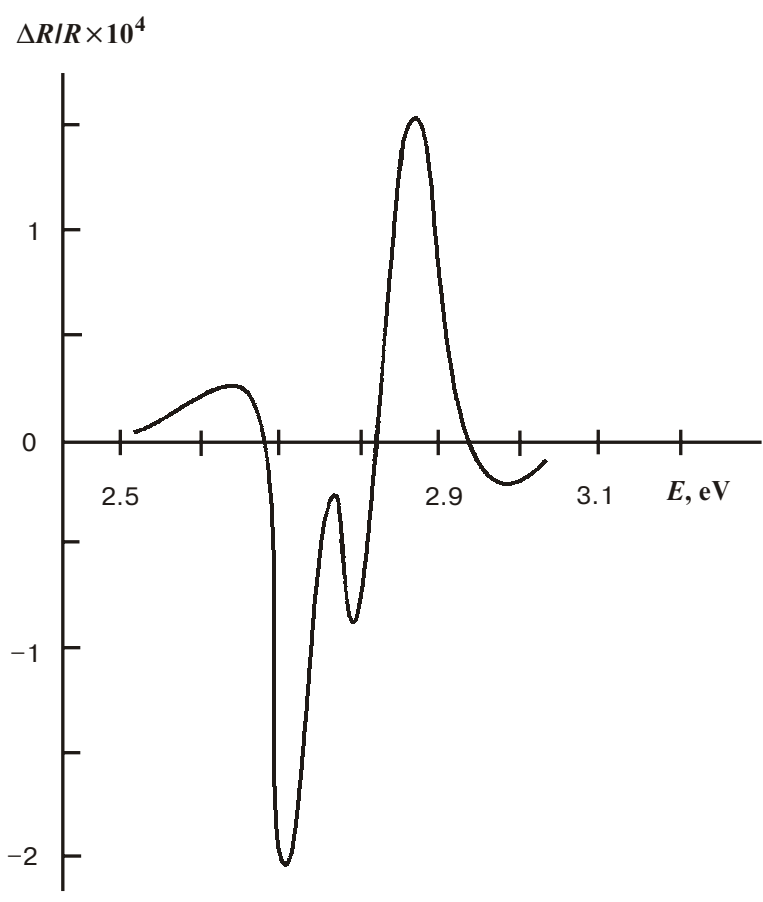

Fig. 2. Electroreflectance spectrum of $n$-GaP film after irradiation by $\gamma$-guanta with the dose $2 \cdot 10^{5}$ rad. The constant bias is $0.2 \mathrm{~V}$, modulating voltages is $0.5 \mathrm{~V}$.

tion-stimulated relaxation of internal mechanical straines. As a consequence, reduced was the scattering of lightexcited charge carriers, and the time of there energy relaxation increased from $4.5 \cdot 10^{-15}$ up to $8.2 \cdot 10^{-15} \mathrm{~s}$.
Unresolved $E_{0}$ and $E_{0}+\Delta_{0}$ electron transitions in initial homoepitaxial GaP films characterized by blurred electroreflectance bands, which is caused by internal mechanical straines in them, were resolved only after radiation-stimulated relaxation of these straines.

The determined value of the spin-orbital splitting $\Delta_{0}=$ $=\Gamma_{8 v}-\Gamma_{7 v}=0.11 \mathrm{eV}$ coincides with the literature data. The obtained results can be used when elaborating a radiation technology for manufacturing new generation devices based on GaP homoepitaxial films.

\section{References}

1. E.F. Venger, L.A. Matveeva // Neorganicheskiye materialy, 33(2), p. 153-157 (1997), in Russian.

2. Yu. A. Tkhorik, Structural relaxation in semiconductor crystals and device structures. "Naukova dumka", Kyiv,1994, 247 p. (in Russian).

3. O.Yu. Borkovskaya, S.A. Grusha, N.L. Dmitruk et al. // Zhurnal tekhnicheskoi fiziki, 55(10), p. 1977-1982 (1985), in Russian.

4. V.A. Tyagai, O.V. Snitko, Light electroreflectance in semiconductors. "Naukova dumka", Kyiv, 1980, 302 p. (in Russian).

5. P. Yu, M. Cardona. Principles of semiconductor physics. "Fizmatlit", Moscow, 2002, 560 p. (in Russian).

6. M. Cardona, K.L. Shaklee, F.H. Pollak // Phys. Rev., 154(3), p. 696-720 (1967).

7. G.L. Bir, G.E. Pikus. Symmetry and deformational effects in semiconductors. "Nauka", Moscow, 1972, 584 p. (in Russian). 